\title{
Free Nano-Object Ramsey Interferometry for Large Quantum Superpositions
}

\author{
C. Wan, ${ }^{1}$ M. Scala, ${ }^{1}$ G. W. Morley, ${ }^{2}$ ATM. A. Rahman,${ }^{2,3}$ H. Ulbricht, ${ }^{4}$ J. Bateman, ${ }^{5}$ \\ P. F. Barker, ${ }^{3}$ S. Bose, ${ }^{3, *}$ and M. S. Kim ${ }^{1}$ \\ ${ }^{1}$ QOLS, Blackett Laboratory, Imperial College London, London SW7 2BW, United Kingdom \\ ${ }^{2}$ Department of Physics, University of Warwick, Gibbet Hill Road, Coventry CV4 7AL, United Kingdom \\ ${ }^{3}$ Department of Physics and Astronomy, University College London, Gower Street, London WCIE 6BT, United Kingdom \\ ${ }^{4}$ Department of Physics and Astronomy, University of Southampton, Southampton SO17 1BJ, United Kingdom \\ ${ }^{5}$ Department of Physics, College of Science, Swansea University, Swansea SA2 8PP, United Kingdom
}

(Received 26 January 2016; published 28 September 2016)

\begin{abstract}
We propose an interferometric scheme based on an untrapped nano-object subjected to gravity. The motion of the center of mass (c.m.) of the free object is coupled to its internal spin system magnetically, and a free flight scheme is developed based on coherent spin control. The wave packet of the test object, under a spin-dependent force, may then be delocalized to a macroscopic scale. A gravity induced dynamical phase (accrued solely on the spin state, and measured through a Ramsey scheme) is used to reveal the above spatially delocalized superposition of the spin-nano-object composite system that arises during our scheme. We find a remarkable immunity to the motional noise in the c.m. (initially in a thermal state with moderate cooling), and also a dynamical decoupling nature of the scheme itself. Together they secure a high visibility of the resulting Ramsey fringes. The mass independence of our scheme makes it viable for a nano-object selected from an ensemble with a high mass variability. Given these advantages, a quantum superposition with a $100 \mathrm{~nm}$ spatial separation for a massive object of $10^{9} \mathrm{amu}$ is achievable experimentally, providing a route to test postulated modifications of quantum theory such as continuous spontaneous localization.
\end{abstract}

DOI: 10.1103/PhysRevLett.117.143003

Introduction.-It is expected by a significant community of researchers that when one reaches a superposition of quantum states separated spatially by $\sim 100 \mathrm{~nm}$ for objects of mass $\sim 10^{9}$ amu or larger, some hitherto unseen modifications of quantum mechanics [1,2] or self-gravitational effects (Schrödinger-Newton equations) [3] may start manifesting. Even practically, such highly nonclassical states will have varied applications in quantum technology such as in metrology. Hence, generating such states, and indeed evidencing them, is of prime importance in the macroscopic frontier of quantum technology. Over the years several proposals for probing spatial superpositions of confined macroscopic objects have been proposed [4-14], but tethering or trapping naturally limits the distance that the superposed state can be separated, and the trapping mechanism itself might offer a route to decoherence. Thus, many recent proposals involve free flight - they have proposed to achieve large spatial superpositions through nonlinear optomechanics using cavity induced measurements [15,16] and through the Talbot intereference of a nano-object ensemble [17]. However, access to strong optomechanical nonlinearities and/or the

Published by the American Physical Society under the terms of the Creative Commons Attribution 3.0 License. Further distribution of this work must maintain attribution to the author(s) and the published article's title, journal citation, and DOI. conditional preparation of superpositions are required in the former set of proposals, while mass dispersion is a difficulty encountered in the latter type of proposals. Here, we thus propose to use Ramsey interferometry of untrapped nano-objects to create and probe superpositions. The scale of the superposition is controllable through the flight time and magnetic field gradients, while the mass does not appear in the relevant interferometric phase.

In this Letter, we propose a scheme based on a free, thermal nano-object with the motion of the center of mass (c.m.) coupled to its internal state. Under coherent control on the internal state the wave packet of the particle could be split and merged in a double-slit interferometry fashion. If, further, the interferometric arms are subjected to different gravitational potentials, a dynamical phase is induced (just as with the neutron interferometry experiments of Ref. [18]) and measured solely on the spin state, which evidences the spatially separated superposition of the test object. The phase itself is independent of the mass so that the nanoparticle ensemble used in the experiment can have a wide range of masses of about the same order of $10^{9} \mathrm{amu}$. With the capability of generating a highly spatially separated superposition and being robust to motional noise, our system paves the way to testing some modifications of quantum theory, such as continuous spontaneous localization (CSL) [19-21].

Model.-As shown in Fig. 1, we first assume that a nanodiamond with a single spin-1 nitrogen-vacancy (NV) 


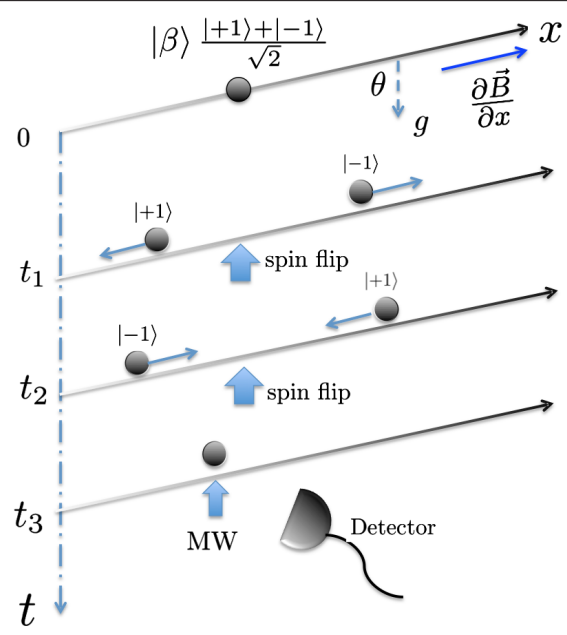

FIG. 1. An untrapped nano-object undergoes an illustrated interferometric scheme. A magnetic field gradient (titled by $\theta$ with respect to gravity) couples the c.m. and the spin of the particle. Starting with a spin state $(|+1\rangle+|-1\rangle) / \sqrt{2}$ at $t=0$, the wave packets of the particle split and accelerate until time $t_{1}$, when a set of microwave (MW) pulses is sent to flip the spin states, which decelerates both wave packet components leading to their motion along the axis reversing after a relevant time. The second set of MW pulses, sent at time $t_{2}$, reverses the direction of acceleration of the separated wave-packet components once again so that after $t_{2}$ they start to decelerate while approaching each other and merge together at $t_{3}$, when a MW pulse is sent to perform the Ramsey measurement.

center is prepared with its c.m. in a low temperature thermal state in a harmonic trap, say, by feedback cooling [22,23]. The NV spin's symmetry axis is aligned with the trapping axis $x$ and with its spin state initialized to $|0\rangle$ (by standard optical pumping). The trapping axis $x$ is tilted by $\theta$ with respect to the direction of the gravitational field and after that a uniform magnetic field gradient $\partial \vec{B} / \partial x$ is introduced, which covers a certain region in the vicinity of the trapped particle and couples its spin and motional degrees of freedom along $x$.

Starting at $t=0$ we release the nano-object and immediately send a microwave (MW) pulse that creates a spin superposition $(|+1\rangle+|-1\rangle) / \sqrt{2}$. The untrapped particle will propagate freely under a spin dependent force and the gravity of the mass; the corresponding Hamiltonian is

$$
H=\frac{\hat{p}^{2}}{2 m}-g_{\mathrm{NV}} \mu_{B} \frac{\partial B}{\partial x} \hat{S}_{z} \hat{x}+m g \cos \theta \hat{x},
$$

where $\mu_{B}$ is the Bohr magneton, $g_{\mathrm{NV}}$ is the Lande $g$ factor, $\theta$ is the tilting angle of the initial trap with respect to the gravitational direction, $g$ is the free fall acceleration, $\hat{S}_{z}$ is the spin $z$ operator of the NV spin, and $\hat{p}$ and $\hat{x}$ are the momentum and position operator along the trapping axis, respectively. We consider the c.m. initially to be an arbitrary coherent state $|\beta\rangle$; under Hamiltonian (1) the particle will propagate in a way that its wave packets spatially separate and accelerate along $x$. The state at time $t$ is then

$$
|\Psi(t)\rangle=\frac{|\psi(t,+1)\rangle|+1\rangle+|\psi(t,-1)\rangle|-1\rangle}{\sqrt{2}},
$$

which is the superposition we aim to demonstrate by the following Ramsey scheme. We flip the spin state of each counterpropagating component at some appropriate times $t_{1}$ and $t_{2}$, by which the split wave packets would merge back after a relevant time, forming a two arm interferometer. The spin flip operation (from $|+1\rangle$ to $|-1\rangle$ or the other way) could be achieved via a two-MW-pulse sequence, provided that the Zeeman splitting due to local magnetic field is comparably large with respect to the MW pulse bandwidth [24]. If the timing of the spin manipulation is controlled by $t_{1}=\frac{1}{3} t_{2}=\frac{1}{4} t_{3}$ we would obtain a separable state at time $t_{3}$. Temporally, the MW pulse timing is precise to $5 \mathrm{~ns}$ or better and the duration of each pulse would be as short as 10 ns. The uncertainty in pulse sequences would in principle result in decoherence on the reduced spin state at the end; however, such an effect would be negligible if the total free flight time is much larger than the pulse times. The state at $t_{3}$ is given by

$$
\left|\Psi\left(t_{3}\right)\right\rangle=\frac{1}{\sqrt{2}}\left|\psi\left(t_{3}\right)\right\rangle\left(|+1\rangle+e^{-i \phi_{g}}|-1\rangle\right),
$$

where $\left|\psi\left(t_{3}\right)\right\rangle$ is the final motional state of the c.m., written in position representation as

$$
\left\langle x \mid \psi\left(t_{3}\right)\right\rangle=e^{-i p_{0} x} e^{-\left[\left(x-x_{0}-p_{0} t_{3} / m-g \cos \theta t_{3}^{2} / 2\right)^{2} / 2\left(\sigma^{\prime}\right)^{2}\right]},
$$

where $p_{0}$ and $x_{0}$ are the initial momentum and position of the nano-object, respectively, and $\sigma^{\prime}$ is the wave packet spread at time $t_{3}$ [24]. By dropping a global phase factor, we have $\phi_{g}=(1 / 16 \hbar) g t_{3}^{3} g_{\mathrm{NV}} \mu_{B}(\partial B / \partial x) \cos \theta$, which is the extra phase stemming from the superposition of spatially separated trajectories subjected to an auxiliary field (local gravity in this case). It could be measured by completing the Ramsey scheme: the second MW pulse on the $\mathrm{NV}$ spin at time $t_{3}$ will map this phase to the population of state $|0\rangle$, whose probability then could be measured by optical fluorescent detection, $P_{0}=\cos ^{2}\left(\phi_{g} / 2\right)=$ $\cos ^{2}\left[(1 / 32 \hbar) g t_{3}^{3} g_{\mathrm{NV}} \mu_{B}(\partial B / \partial x) \cos \theta\right]$. Practically, the particle will be retrapped for a repeated measurement and either $\theta$ or $t_{3}$ would be used as a controllable parameter that shifts the value of $\phi_{g}$, with respect to which a fringe of $P_{0}$ is resolved.

Thermal state.-Remarkably, the phase $\phi_{g}$ is independent of the initial motional condition, featuring an immunity to the initial motional noise in our scheme; consider an initial motional state $\rho_{\text {th }}=\int_{\beta} d^{2} \beta P_{\text {th }}(\beta)|\beta\rangle\langle\beta|$, where $P_{\text {th }}$ is the Glauber $P$ representation for the thermal state. The spin is initialized to $|0\rangle$ in the trap so that it is decoupled from the motion, and as soon as the particle is released, $|0\rangle$ is 
converted to $(|+1\rangle+|-1\rangle) / \sqrt{2}$. Then, at time $t=t_{3}$ we have

$$
\begin{aligned}
\rho_{\mathrm{th}}\left(t_{3}\right)= & \frac{1}{2} \int_{\beta} d \beta P_{\mathrm{th}}(\beta)\left|\psi\left(t_{3}\right)\right\rangle_{\beta}\left\langle\left.\psi\left(t_{3}\right)\right|_{\beta}\right. \\
& \otimes\left(|+1\rangle+e^{i \phi_{g}}|-1\rangle\right)\left(\langle+1|+e^{-i \phi_{g}}\langle-1|\right) .
\end{aligned}
$$

Obviously, the state of the composite system is again factorizable (separable), so the phase difference accrued by the spin states is not affected by the initial thermal motion. A feedback cooling on the initial state of the c.m. to millikelvin temperatures [22] (by which the harmonic potential could barely sustain the thermal excitations) will suffice. This factorizability despite the untrapped motion (which naturally gives rise to dispersion) is a nontrivial feature of our scheme.

Experiment parameters.-The maximum spatial separation $\Delta x_{M}$ of the superposed components is given at half the propagation time $t_{3}$ by

$$
\Delta x_{M}=2 \times \frac{1}{2 m} g_{\mathrm{NV}} \mu_{B} \frac{\partial B}{\partial x}\left(t_{3} / 4\right)^{2} ;
$$

we now analyze the achievable scale of magnitude of this separation under realistic parameters. We consider a diamond sphere of radius $R \sim 100 \mathrm{~nm}$ and with a density of $3500 \mathrm{~kg} / \mathrm{m}^{3}$, whose mass is then $\sim 1.25 \times 10^{-17} \mathrm{~kg}$ (corresponding to $7.5 \times 10^{9} \mathrm{amu}$ ). The coherent evolution time $t_{3}$ is limited by the coherence time of the system accounting for all possible detrimental effects, which will be discussed below, and here we suppose a realistic value of $\sim 100 \mu \mathrm{s}$. Under a field gradient of $(\partial B / \partial x) \sim 10^{7} \mathrm{~T} / \mathrm{m}[26,27]$ we immediately obtain a separation of $\Delta x_{M} \sim 100 \mathrm{~nm}$. Interestingly, this is comparable to the size of the test nano-object. So a good position measurement at time $t_{3} / 2$, such as those used in feedback cooling [22], can even discriminate the two components of the superposition spatially. Of course, this measurement will destroy the superposition so that the superposition has to be tested through the $\phi_{g}$ induced fringes in other runs of the experiment where the measurements are only done at $t_{3}$. Nonetheless, some runs of the experiment measuring the spatial position at time $t_{3} / 2$ will confirm the picture that the components superposed are indeed spatially separated by $100 \mathrm{~nm}$.

Decoherence.-Collisional and thermal decoherence are mostly considered in matter wave interferometry and optomechanical systems [28,29], which can be seen as random momentum kicks during the propagation of the matter wave and whose microscopic description is given by the master equation [17]

$$
\mathcal{L}_{i}(\rho)=\int d \omega \gamma_{i}(\omega) \int_{|n|=1} \frac{d n^{2}}{4 \pi}\left[e^{\left(i \omega n_{x} / c\right) \hat{x}} \rho e^{\left(-i \omega n_{x} / c\right) \hat{x}}-\rho\right],
$$

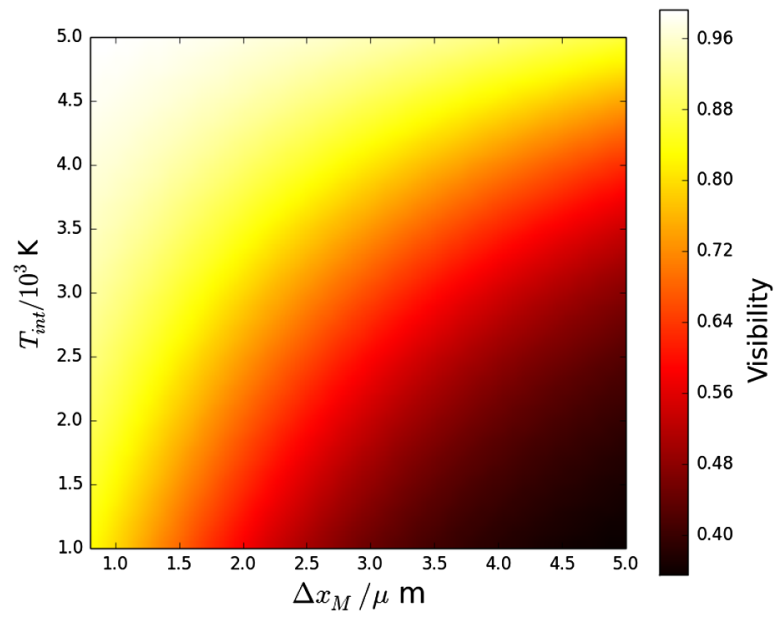

FIG. 2. Estimation on motional decoherence: $\Delta x_{M}$ is the maximum spatial separation and $T_{\text {int }}$ is the internal temperature of the test object. A large high visibility window indicates the strong robustness of our scheme against motional noise.

where $i$ indicates the specific decoherence class, including collisions with residual gas particles, the scattering and absorption of blackbody photons, and the thermal emission of radiation. $\gamma_{i}$ is the spectral rate and $\mathbf{n}$ is the direction cosine of the random momentum kick. Given $\gamma_{i}$ from realistic data the above master equation could be numerically simulated together with the unitary part of the free propagation (acceleration). Because of the entanglement between the spin and mechanical states, the motional decoherence process, specifically the part of it that carries the which-path information of the two counterpropagated wave packets, would eliminate the coherence of the c.m. and the reduced spin system at the end, which subsequently reduces the visibility of the following Ramsey measurement. Practically, the collisional decoherence is suppressed by preparing the system in a high vacuum chamber. As trapping is lifted during the flight the photonic scattering is absent, leaving only the radiative decoherence from the background and the black body radiation of the particle [17]. Here, we provide a theoretical estimation of the upper bound of the detrimental effect from radiative decoherence by considering the worst scenario in the evolution [24]. The resultant interferometric visibility (square modulus of the off-diagonal term of the reduced density matrix of the spin system) of the Ramsey measurement is shown in Fig. 2. The spin dephasing of the NV center will be the last detrimental effect that limits the absolute coherence time of the system. NV centers in isotopically purified bulk diamond can have an electron spin coherence time $T_{2}$ up to $\sim 2 \mathrm{~ms}$ at room temperature [30], but such exceptional times have not been found in nanodiamonds. In order to achieve the longest $T_{2}$, nanodiamonds are made from high purity bulk material with a low density of nitrogen impurities and ${ }^{13} \mathrm{C}$. Nanodiamond pillars with a 300-500 nm diameter have shown a spin echo $T_{2}$ time 
of over $300 \mu \mathrm{s}$ [31]. Pillars with a $50 \mathrm{~nm}$ diameter and $150 \mathrm{~nm}$ length have achieved a spin echo $T_{2}$ time of $79 \mu \mathrm{s}$ [32]. This time was further extended by appropriate decoupling techniques. Interestingly, as an additional advantage, the sequence of MW pulses applied in our scheme, namely, $(\pi / 2)_{x},(\pi)_{x},(\pi)_{x},(\pi / 2)_{x}$, is a dynamical decoupling sequence [33] that would echo out the noise attributed to any slow and spin relevant effect such as a quasistatic spin bath. More interestingly, the perturbation from the rotational degrees of freedom, induced by an unknown torque on the particle since the NV center is not necessarily situated at the c.m. of the nanodiamond, could also be suppressed by virtue of this technique [24].

Testing spontaneous collapse models.-Using the macroscopicity measure $\mu$ in Ref. [34], a high visibility of our interferometry would impose a value of $\mu=24$ for our system, which is comparable to the largest among the proposed experiments to date, such as those employing oscillating micromirrors or larger molecules. Since macroscopicity is intimately connected to the testability of any macrorealistic modification of quantum theory; in this regard, another key purpose for the creation of the spatially large superposition will be to test the CSL model [19-21], which is characterized by the localization length $r_{\mathrm{CSL}}$ and rate $\lambda_{\mathrm{CSL}}$. The former is about $100 \mathrm{~nm}$, which sets the scale above which the delocalized matter wave gets localized. The latter represents the average collapse rate at one proton mass, on which the interferometric experiment could place a bound. For our scheme, if we were to observe a high visibility (as expected from the above considerations of environmental decoherence), it would bound the collapse rate to $[1]$

$$
\lambda \leq 1 / 2 N^{2} t_{3} \sim 10^{-14} \mathrm{~s}^{-1},
$$

where $N$ is the number of protons of our test object, which is $10^{9}$ in our case. The version of CSL by Adler $\left(\lambda \sim 10^{-9} \mathrm{~s}^{-1}\right)$ [21] should thus already decohere our superposition by a mechanism beyond standard quantum theory, while, to access the version by Ghirardi-RiminiWeber [35] $\left(\lambda \sim 10^{-16} \mathrm{~s}^{-1}\right)$ one will need to extend the coherence time of the NV center spin by 2 orders of magnitude, which is challenging.

Other intrinsic decoherence.-In order to unambiguously test CSL, it is crucial to rule out the significance of other hypothetical localization effects in the mescoscopic region we are considering. For instance, the gravitational time dilation effect [36], which couples the internal degree of freedom to the c.m. motion of a compound system when the state of the latter is spatially separated in the direction of a gravitational field, will induce a dephasing process on a c.m. subsystem. Substituting the relevant parameters of our model $\left(T_{\text {int }}=400 \mathrm{~K}, \Delta x=100 \mathrm{~nm}\right.$, and $\left.N=10^{9}\right)$ we immediately obtain a coherence time admitted by this time dilation effect of $1000 \mathrm{~s}$, which is sufficiently far from the scale of the coherence time we consider. In a similar vein, if we consider gravitational reduction models [37], then, assuming the mass density to be concentrated around the nuclei [38], we obtain a decoherence time of $100 \mathrm{~s}$. Moreover, by engineering a superposition of distinct kinetic energy states by changing the initial spin state to $(|0\rangle+|+1\rangle) / \sqrt{2}$ in our free-flight scheme, we can constrain an effective parameter $\Theta$ of space-time textures [39] to $\lesssim 10^{25}$ contingent on a high interferometric visibility.

Multiple NVs.-Diamond samples with multi NVs are easy to obtain and provide a large spin-dependent fluorescence increasing the sensitivity of the final spin measurement. It has been experimentally demonstrated that the orientations of all those NV centers' axes could be identically aligned to one of the four possible directions in the diamond crystal and their spin states could also be collectively manipulated and measured with Ramsey pulses [40-42]. The mechanism in this multi-NV scenario will follow the similar formula developed above [24]; starting with an arbitrary coherent state for the c.m. and an $l$ fold product state of $(|+1\rangle+|-1\rangle) / \sqrt{2}$ for the spin ensemble, the composite system ends up again a separable state in which the spin state is trivially an $l$ fold product state of $\left(|+1\rangle+e^{-i \phi_{g}}|-1\rangle\right) / \sqrt{2}$. Evidencing this accrued phase on the multispin ensemble would reveal the superposition of the intermediate state of the corresponding collective spin-c.m. system.

Conclusions. - We have shown a method to generate and evidence superpositions of two c.m. states of a free (in the sense of being untrapped) nano-object of $\sim 10^{9}$ amu mass. The untrapped nature of the particle, in conjunction with spin dependent acceleration or deceleration in an external magnetic field gradient enables us to reach $100 \mathrm{~nm}$ spatial separations between the superposed components. This can open up possibilities of testing some of the spontaneous collapse models such as Adler's model [21] through a method that is qualitatively very different from the recently proposed noninterferometric tests [43]. The scheme completely surpasses the scale of the spatial separation possible through a trapped particle of the same mass [44] by $10^{3}$ orders of magnitude (essentially due to the absence of a finite frequency). In comparison to the adaptation of the Ramsey-Bordé technique to nano-objects [45], we have employed a state-dependent force that significantly boosts the delocalization scale of the matter wave. Such a macroscopicity is unattainable via photonic momentum kicks in the Ramsey-Bordé method, and the concomitant Doppler dephasing is negligible in our NV case [24]. A positive feature is that the relevant interferometric phase can be probed solely via spin Ramsey interferometry without directly measuring the matter wave distribution $[17,46]$. Moreover, from the point of view of control, an electron spin in a solid is a promising system with lower noise compared to optical frequency fields in cavity optomechanics, while its coupling to the c.m. through a magnetic field gradient could potentially be easier than achieving 
strong optomechanical couplings. Uniquely, the MW control is also naturally a dynamical decoupling that suppresses those slow detrimental dynamics, so that the best coherence times of $100 \mu \mathrm{s}$ can be used. The fact that the scale of spatial separation can be increased substantially by using untrapped particles, and yet be evidenced solely by a spin-only Ramsey interferometry in a gravitational potential, and indeed be independent of both the initial thermal state of the nano-object and its mass, greatly facilitates the possibility of the interferometric probing of large superpositions. In view of the fact that the manipulation of a spin-full levitated nano-object is being intensely pursued experimentally [23] at the moment, our scheme should be realizable in the near future.

We acknowledge EPSRC Grant No. EP/J014664/1. This work was also supported by EPSRC as part of the UK Hub in Networked Quantum Information Technologies (NQIT), Grant No. EP/M013243/1. G. W. M. is supported by the Royal Society. H. U. acknowledges support by the John F Templeton Foundation (Grant No. 39530) and the Foundational Questions Institute (FQXi). C. W. is supported by an Imperial CSC scholarship. M. S. K. acknowledges support by a Leverhulme Trust Research Grant (No. RPG-2014-055).

*Corresponding Author.

s.bose@ucl.ac.uk

[1] A. Bassi, K. Lochan, S. Satin, T. P. Singh, and H. Ulbricht, Rev. Mod. Phys. 85, 471 (2013).

[2] S. L. Adler and A. Bassi, J. Phys. A 40, 15083 (2007).

[3] M. Bahrami, A. Grosshardt, A. Donadi, and A. Bassi, New J. Phys. 16, 115007 (2014).

[4] S. Bose, K. Jacobs, and P. L. Knight, Phys. Rev. A 59, 3204 (1999).

[5] A. D. Armour, M. P. Blencowe, and K. C. Schwab, Phys. Rev. Lett. 88, 148301 (2002).

[6] W. Marshall, C. Simon, R. Penrose, and D. Bouwmeester, Phys. Rev. Lett. 91, 130401 (2003).

[7] P. Rabl, P. Cappellaro, M. V. Gurudev Dutt, L. Jiang, J. R. Maze, and M.D. Lukin, Phys. Rev. B 79, 041302(R) (2009).

[8] O. Romero-Isart, M. L. Juan, R. Quidant, and J. I. Cirac, New J. Phys. 12, 033015 (2010).

[9] P. F. Barker and M. N. Shneider, Phys. Rev. A 81, 023826 (2010).

[10] D. E. Chang, C. A. Regal, S. B. Papp, D. J. Wilson, J. Ye, O. Painter, H. J. Kimble, and P. Zoller, Proc. Natl. Acad. Sci. U.S.A. 107, 1005 (2010).

[11] S. Gerlich, S. Eibenberger, M. Tomandl, S. Nimmrichter, K. Hornberger, P. J. Fagan, J. Tüxen, M. Mayor, and M. Arndt, Nat. Commun. 2, 263 (2011).

[12] O. Romero-Isart, L. Clemente, C. Navau, A. Sanchez, and J. I. Cirac, Phys. Rev. Lett. 109, 147205 (2012).

[13] M. Cirio, G. K. Brennen, and J. Twamley, Phys. Rev. Lett. 109, 147206 (2012)
[14] Z.-q. Yin, T. Li, X. Zhang, and L. M. Duan, Phys. Rev. A 88, 033614 (2013).

[15] O. Romero-Isart, A. C. Pflanzer, F. Blaser, R. Kaltenbaek, N. Kiesel, M. Aspelmeyer, and J. I. Cirac, Phys. Rev. Lett. 107, 020405 (2011).

[16] O. Romero-Isart, Phys. Rev. A 84, 052121 (2011).

[17] J. Bateman, S. Nimmrichter, K. Hornberger, and $H$. Ulbricht, Nat. Commun. 5, 4788 (2014).

[18] A. W. Overhauser and R. Colella, Phys. Rev. Lett. 33, 1237 (1974); R. Collela, A. W. Overhauser, and S. A. Werner, Phys. Rev. Lett. 34, 1472 (1975); S. A. Werner, J. L. Staudenmann, and R. Colella, Phys. Rev. Lett. 42, 1103 (1979).

[19] P. Pearle, Phys. Rev. A 39, 2277 (1989).

[20] S. L. Adler and A. Bassi, J. Phys. A 40, 15083 (2007).

[21] S. L. Alder, J. Phys. A 40, 2935 (2007).

[22] T. Li, S. Kheifets, and M. G. Raizen, Nat. Phys. 7, 527 (2011); J. Gieseler, B. Deutsch, R. Quidant, and L. Novotny, Phys. Rev. Lett. 109, 103603 (2012).

[23] A. Kuhlicke, A. W. Schell, J. Zoll, and O. Benson, Appl. Phys. Lett. 105, 073101 (2014); L. O. Neukirch, E. V. Harrtman, J. M. Rosenholm, and A. N. Vamivakas, Nat. Photonics 9, 653 (2015); K. Hammerer and M. Aspelmeyer, Nat. Photonics 9, 633 (2015); J. Millen, P. Z. G. Fonseca, T. Mavrogordatos, T. S. Monteiro, and P. F. Barker, Phys. Rev. Lett. 114, 123602 (2015); T. M. Hoang, J. Ahsn, J. Bang, and T. Li, Nat. Commun. 7, 12250 (2016); A. T. M. A. Rahman, A. C. Frangeskou, M. S. Kim, S. Bose, G. W. Morley \& P. F. Barker, Sci. Rep. 6, 21633 (2016).

[24] See Supplemental Material at http://link.aps.org/ supplemental/10.1103/PhysRevLett.117.143003, which provides further details for the calculation of the scheme and the decoherence process, the experimental design and some other systematic effect analysis, and Ref. [25] is included.

[25] R. Schirhagl, K. Chang, M. Loretz, and C. L. Degen, Annu. Rev. Phys. Chem. 65, 83 (2014).

[26] H. J. Mamin, M. Poggio, C. L. Degen, and D. Rugar, Nat. Nanotechnol. 2, 301 (2007).

[27] C. Tsang et al., IEEE Trans. Magn. 42, 145 (2006).

[28] K. Hornberger, J. E. Sipe, and M. Arndt, Phys. Rev. A 70, 053608 (2004).

[29] L. Hackerüller, K. Hornberger, B. Brezger, A. Zeilinger, and M. Arndt, Nature (London) 427, 711 (2004).

[30] G. Balasubramanian et al., Nat. Mater. 8, 383 (2009).

[31] P. Andrich, B. J. Alemán, J. C. Lee, K. Ohno, C. F. de las Casas, F. J. Heremans, E. L. Hu, and D. D. Awschalom, Nano Lett. 14, 4959 (2014).

[32] M. E. Trusheim, L. Li, A. Laraoui, E. H. Chen, H. Bakhru, T. Schröder, O. Gaathon, C. A. Meriles, and D. Englund, Nano Lett. 14, 32 (2014).

[33] C. P. Slichter, Principles of Magnetic Resonance (Springer Press, New York, 1996).

[34] S. Nimmrichter and K. Hornberger, Phys. Rev. Lett. 110, 160403 (2013).

[35] G. C. Ghirardi, A. Rimini, and T. Weber, Phys. Rev. D 34, 470 (1986).

[36] I. Pikovski, M. Zych, F. Costa, and C. Brukner, Nat. Phys. 11, 668 (2015).

[37] R. Penrose, Gen. Relativ. Gravit. 28, 581 (1996); L. Diósi, Phys. Rev. 40, 1165 (1989). 
[38] D. Kleckner, I. Pikovski, E. Jeffrey, L. Ament, E. Eliel, J. van den Brink, and D. Bouwmeester, New J. Phys. 10, 095020 (2008).

[39] C. Anastopoulos and B.-L. Hu, Classical Quantum Gravity 30, 165007 (2013).

[40] A. M. Edmonds, U. F. S. DHaenens-Johansson, R. J. Cruddace, M. E. Newton, K. M. C. Fu, C. Santori, R. G. Beausoleil, D. J. Twitchen, and M. L. Markham, Phys. Rev. B 86, 035201 (2012).

[41] M. Lesik, J.-P. Tetienne, A. Tallaire, J. Achard, V. Mille, A. Gicquel, J.-F. Roch, and V. Jacques, Appl. Phys. Lett. 104, 113107 (2014).

[42] J. Michl et al., Appl. Phys. Lett. 104, 102407 (2014).

[43] M. Bahrami, M. Paternostro, A. Bassi, and H. Ulbricht, Phys. Rev. Lett. 112, 210404 (2014); S. Nimmrichter,
K. Hornberger, and K. Hammerer, Phys. Rev. Lett. 113, 020405 (2014); L. Disi, Phys. Rev. Lett. 114, 050403 (2015); D. Goldwater, M. Paterostro, and P. F. Barker, Phys. Rev. A 94, 010104 (2016); J. Li, S. Zippilli, J. Zhang, and D. Vitali, Phys. Rev. A 93, 050102(R) (2016).

[44] M. Scala, M. S. Kim, G. W. Morley, P. F. Barker, and S. Bose, Phys. Rev. Lett. 111, 180403 (2013); C. Wan, M. Scala, S. Bose, A. C. Frangeskou, A. T. M. A. Rahman, G. W. Morley, P. F. Barker and M. S. Kim, Phys. Rev. A 93, 043852 (2016).

[45] A. Albrecht, A. Retzker, and M. B. Plenio, Phys. Rev. A 90, 033834 (2014).

[46] S. Nimmrichter, K. Hornberger, P. Haslinger, and M. Arndt, Phys. Rev. A 83, 043621 (2011). 